\title{
Doença de Parkinson e exercício físico
}

\author{
Parkinson's Disease and physical exercise
}

\section{Vanessa de Araújo Rubert', Diogo Cunha dos Reis ${ }^{2}$, Audrey Cristine Esteves ${ }^{3}$}

\section{RESUMO}

A Doença de Parkinson (DP) é descrita como uma desordem neurodegenerativa que afeta a população idosa, a qual compromete os neurônios dopaminérgicos da substância negra, culminando no déficit de dopamina no corpo estriado influenciando na modulação do movimento. Entre os sintomas encontrados em pacientes com DP destacam-se: acinesia ou bradicinesia, rigidez, tremor de repouso, instabilidade postural, em alguns casos existe também comprometimento de ordem cognitiva, afetiva e autonômica. Sendo assim, pretende-se com este estudo verificar na literatura os benefícios do exercício físico $(\mathrm{EF})$ para indivíduos com DP. Com este estudo, pôde-se observar que existem poucos trabalhos na literatura enfocando esta temática, mas parece haver uma tendência em acreditar que o exercício físico regular (aeróbico) é benéfico para pacientes com DP, pois reduz sintomas como a hipocinesia, bradicinesia, distúrbios da marcha, degeneração neuronal, sendo então reconhecido como uma ferramenta que auxilia a terapia medicamentosa.

\section{Unitermos: Doença de Parkinson. Exercício Físico. Trans-} tornos Motores.

Citação: Rubert VA, Reis DC, Esteves AC. Doença de Parkinson e exercício físico.

\section{SUMMARY}

The Parkinson's disease (PD) is described as a neurodegenerative illness which affects the old aged population, which compromises the dopamine neurons of the substantia nigra, culminating in the dopamine deficit in the striated body - structures that are part of the Central Nervous System - influencing in the modulation of the movement. Among the symptoms found in the PD are distinguished: akinesia or bradikinesia, rigidity, rest tremor, postural instability, in some cases can also occur implication of cognitive order, affective and autonomic. In such case, it is intended with this study to verify in literature the benefits of the physical exercise (PE) for people with PD. With this study, it could be observed that there are few papers in the literature focusing this thematic, but it seems to have a trend in believing that the regular physical exercise (aerobic exercise) is well accepted for patients with DP and being recognized as an auxiliary mean to traditional therapies (withmedication).

Keywords: Parkinson Disease. Exercise. Movement Disorders.

Citation: Rubert VA, Reis DG, Esteves AC. Parkinson's Disease andphysical exercise.

Endereço para correspondência: Diogo Cunha dos Reis Laboratório de Biomecânica - Centro de Desportos - Universidade Federal de Santa Catarina - Campus Universitário - Trindade - CEP 88040-900 - Florianópolis - SG - Brasil. Fone: (48) 37218530

E-mail: diogo.biomecanica@gmail.com

Recebido em: 10/03/06 Revisão: 11/03/06 a 05/09/06

Aceito em: 06/09/06

Conflito de interesses: não 


\section{INTRODUÇÃO}

A doença de Parkinson (DP) é uma doença degenerativa do sistema nervoso central, com implicações profundas para o indivíduo, causando principalmente déficits nas funções motoras, mas também poderá ser responsável por outras manifestações sistêmicas associadas e nas funções autônomas ${ }^{1,2}$.

A DP está entre as doenças neuro-degenerativas de maior incidência em pessoas idosas ${ }^{3}$. Aproximadamente, $0,1 \%$ da população geral e $1 \%$ da população acima de 65 anos é acometida por essa síndrome, sendo deste apenas um pequeno número de pessoas sofrem de demências ${ }^{4,5}$.

A DP foi descrita pela primeira vez em 1817, por James Parkinson. Através de observações e exames clínicos, Parkinson descreveu sintomas que são até a atualidade aceitos como as principais manifestações da DP: tremor, rigidez muscular, acinesia, anomalias posturais e anormalidades na marcha ${ }^{1}$.

Conforme o autor citado, a etiologia do $\mathrm{Pa}$ rkinson tem sido controversa, associa-se à genética, ao estresse, exposição a ambientes tóxicos e infecciosos. Há uma tendência em acreditar que a morte do tecido neural é causada pelo estresse oxidativo, devido à redução de glutation, um sistema antioxidante importante capaz de neutralizar os radicais livres, mesmo assim, por enquanto não se tem uma causa definida para essa degeneração ${ }^{6}$.

Em exames neurológicos podem ser observadas perdas extensas de células do mesencéfalo, conhecidas como substância negra pars compacta $(\mathrm{SNc})^{3,7}$. Esta diminuição celular leva a crer que a DP é causada por um colapso na comunicação entre a SNc e o striatum, dessa sinapse resulta a liberação de dopamina ${ }^{3}$. A perda de neurônios da $\mathrm{SNc}$ causa um esgotamento de dopamina nos gânglios de base o que causa alterações na elaboração de acetilcolina ${ }^{1,8}$. A deficiência de dopamina produz um grave efeito no sistema extrapiramidal resultando em déficits na coordenação muscular e nas atividades musculares, com isso resultando em problemas na manutenção da postura, alterações na marcha, coordenação fina, enrijecimento muscular e outros ${ }^{9-12}$.

Devido à progressão da DP, em 1967, Hoehn e Yahr classificaram-na em estágios, que representam o grau de dificuldade do paciente, variando de I a V. Estágio I: sinais e sintomas em um lado do corpo, leves e inconvenientes, porém não incapacitantes, usualmente presença de tremor em um membro; estágio II: sintomas bilaterais, disfunção mínima, comprometimento da postura e marcha; estágio
III: lentidão significativa dos movimentos corporais, disfunção do equilíbrio de marcha e ortostático, disfunção generalizada moderadamente grave, estágio IV e V: sintomas graves, locomove-se por uma distância limitada, rigidez e bradicinesia, perda total da independência, respostas imprecisas a levodopa e doenças neuropsiquiátricas ${ }^{13}$.

A Levodopa é a principal droga utilizada para a DP, entretanto esse tratamento tem inúmeras desvantagens, pois ela não contém a progressão da doença, apenas ameniza os sintomas. No decorrer do tratamento a droga perde sua eficácia e muitos efeitos colaterais sérios podem ocorrer tal como a acinesia, sintomas de psicoses, aumento do tempo na fase sem medicamento, hipocinesia e outros sintomas.

Considerando esses efeitos, pondera-se a hipótese que a prática regular de exercícios físicos $(\mathrm{EF})$ poderá beneficiar as pessoas acometidas pelo Parkinson, se não responsável pela cura, mas tendo papel importante para amenizar ou retardar o aparecimento dos sintomas e garantir alguma independência para os parkinsonianos.

Assim, pretende-se com este estudo verificar os efeitos do exercício físico para portadores de DP, por meio de revisão bibliográfica.

\section{MÉTODO}

As fontes de dados pesquisadas consistiram-se basicamente de artigos científicos publicados em periódicos internacionais disponíveis on-line no portal Periódicos da Capes (http://www.periodicos.capes. gov.br), no qual foram pesquisadas as seguintes palavras-chave: Doença de Parkinson e exercício físico. As consultas se limitaram à produção científica dos últimos dez anos, publicadas nos idiomas português e inglês.

Os dados foram comparados com a intenção de verificar as tendências dos estudos bem como os resultados reportados por estes em relação à adoção de programas de exercícios físicos em pacientes com DP em diversos estágios.

Procurou-se dar ênfase nas discussões sobre as alterações das respostas sintomáticas do quadro clínico do paciente durante o período de realização das atividades físicas bem como a melhoria da qualidade de vida do paciente.

\section{RESULTADOS E DISCUSSÃO}

Na Tabela 1 encontram-se especificados os artigos utilizados na discussão da dos efeitos do EF nos portadores da DP, acompanhados por uma breve descrição destes. 
Tabela 1. Descrição dos estudos encontrados que tratam dos efeitos dos exercícios físicos nos portadores da Doença de Parkinson.

\begin{tabular}{|c|c|c|c|}
\hline AUTOR, ANO & NÚMERO DE PACIENTES & DESCRIÇÃO DO ESTUDO & CONCLUSÕES \\
\hline Canning et al, $1997^{19}$ & $\begin{array}{l}13 \text { homens e } 3 \text { mulheres } \\
\text { portadores da DP. }\end{array}$ & $\begin{array}{l}\text { Avaliação do Vo2 máximo, função respi- } \\
\text { ratória e marcha em portadores da DP } \\
\text { moderadamente ativos e sedentários. }\end{array}$ & $\begin{array}{l}\text { Portadores da DP sedentários apresentaram ní- } \\
\text { veis de Vo2 máximo abaixo dos moderadamen- } \\
\text { te ativos e dos não portadores, demonstrando } \\
\text { que em indivíduos com leve a moderada DP os } \\
\text { exercícios aeróbicos regulares mantém normal } \\
\text { sua capacidade cardiovascular apesar do déficit } \\
\text { neurológico. }\end{array}$ \\
\hline Lökk, $2000^{13}$ & $\begin{array}{l}11 \text { homens e } 8 \text { mulheres } \\
\text { portadores da DP. }\end{array}$ & $\begin{array}{l}\text { Efeitos de uma semana de caminhada } \\
\text { em terreno montanhoso nas capacidades } \\
\text { funcionais. }\end{array}$ & $\begin{array}{l}\text { São registradas melhoras gerais nos pacientes } \\
\text { ao fim da semana de treinamento, porém estas } \\
\text { não são mantidas após } 18 \text { semanas. }\end{array}$ \\
\hline Miyai et al, $2000^{15}$ & $\begin{array}{l}5 \text { homens e } 5 \text { mulheres porta- } \\
\text { dores da DP. }\end{array}$ & $\begin{array}{l}4 \text { semanas de treinamento de marcha } \\
\text { em esteira, apoiando frações progressi- } \\
\text { vas do peso corporal. }\end{array}$ & $\begin{array}{l}\text { Observaram-se melhorias significativas para a } \\
\text { performance motora, para a deambulação e } \\
\text { para as atividades da vida diária (AVD). Resul- } \\
\text { tados melhores que os da fisioterapia comum. }\end{array}$ \\
\hline Hirsch et al, $2003^{18}$ & 15 portadores da DP. & $\begin{array}{l}\text { Comparação dos efeitos de um progra- } \\
\text { ma de treinamento de equilíbrio e um } \\
\text { programa combinando equilíbrio e resis- } \\
\text { tência muscular de membros inferiores, } \\
\text { após } 10 \text { semanas de intervenção. }\end{array}$ & $\begin{array}{l}\text { Ambos os tipos de treinamento melhoraram } \\
\text { o equilíbrio e aumentaram os níveis de força } \\
\text { muscular localizada, porém os ganhos foram } \\
\text { maiores para o programa combinado. Esses } \\
\text { ganhos persistiram durante pelo menos } 4 \\
\text { semanas. }\end{array}$ \\
\hline Pohl et al, $2003^{17}$ & $\begin{array}{l}12 \text { homens e } 5 \text { mulheres } \\
\text { portadores da DP. }\end{array}$ & $\begin{array}{l}\text { Comparação dos efeitos de um trei- } \\
\text { namento de marcha em esteira com } \\
\text { apoio progressivo do peso do corpo com } \\
\text { o método de reabilitação da marcha } \\
\text { através da facilitação neuromuscular } \\
\text { proprioceptiva, após } 4 \text { dias de interven- } \\
\text { ção controlada. }\end{array}$ & $\begin{array}{l}\text { Observou-se uma melhora significativa na } \\
\text { velocidade e comprimento do passo depois do } \\
\text { treinamento da marcha na esteira, mostrando } \\
\text { uma redução relevante na fase de duplo apoio } \\
\text { da marcha e nenhuma mudança significativa } \\
\text { depois do FNP tradicional. }\end{array}$ \\
\hline $\begin{array}{l}\text { Smith e Zigmond, } \\
200314 .\end{array}$ & Revisão. & $\begin{array}{l}\text { Revisão de estudos sobre os resultados } \\
\text { dos exercícios físicos sobre a recuperação } \\
\text { funcional do cérebro. }\end{array}$ & $\begin{array}{l}\text { A atividade física pode funcionar reduzindo } \\
\text { a degeneração de neurônios dopaminérgicos } \\
\text { e o desenvolvimento dos sintomas da DP nos } \\
\text { pacientes. }\end{array}$ \\
\hline $\begin{array}{l}\text { Sutoo e Akiyama, } \\
200320 .\end{array}$ & Revisão. & $\begin{array}{l}\text { Revisão de estudos sobre os efeitos dos } \\
\text { exercícios físicos nos sintomas da DP. }\end{array}$ & $\begin{array}{l}\text { Alguns sintomas da DP podem ser melhorados } \\
\text { através de exercícios físicos. }\end{array}$ \\
\hline
\end{tabular}

Foram encontrados poucos estudos que façam uma relação direta entre o EF e a DP, contudo observou-se uma tendência em acreditar que o EF é capaz de amenizar os efeitos do desuso assim como alguns sintomas da doença (hipocinesia, bradicinesia, distúrbios da marcha, degeneração neuronal).

No início do quadro de Parkinson a atividade fisica pode funcionar reduzindo a degeneração de neurônios dopaminérgicos e o desenvolvimento dos sintomas da DP nos pacientes ${ }^{14}$. Logo no inicio do desenvolvimento da doença, pacientes podem aprender a realizar estratégias de comportamento alternativas que irão conduzir para menor comprometimento do sistema motor, mudança essa que é freqüentemente observada em modelos animais, que ao serem forçados a utilizar o membro com características iniciais da doença, apresentaram evolução mais lenta dos sintomas.
Em um estudo, foram testados 10 pacientes com DP por quatro semanas com o objetivo de comparar os efeitos da fisioterapia convencional com um treinamento de caminhada apoiando frações progressivas (treadmill) do peso do corpo $^{15}$. Os autores sugerem que seções de 45 minutos três vezes por semana de caminhadas com a sustentação parcial do peso do corpo, além de ser um trabalho aeróbico para essas pessoas, apresentam melhoras significativas para a amplitude e velocidade da passada, qualidades essas que agem na melhoria e estabilidade da marcha. Assim, para pessoas com DP o treinamento de passadas com apoio parcial do peso do corpo, produz mais melhorias significativas para a performance motora, para a deambulação e para as atividades da vida diária (AVD) que a fisioterapia comum. 
Em outro, comparando a fisioterapia tradicional com este outro método, foi verificado que esse tipo de atividade tem um efeito duradouro na marcha dos pacientes com Parkinson agindo principalmente sobre o arrastar dos pés característicos desses pacientes e no número de passos ${ }^{16}$. Os autores ainda sugerem que essa prática aumenta o tempo de ação do medicamento.

O treadmill tem sido uma promissora terapia no processo de reabilitação de pacientes com anomalias na marcha e mais recentemente tem sido utilizado em pacientes com DP e resultando em melhoras nos parâmetros de marcha maiores que as terapias convencionais ${ }^{17}$.

Os autores citam ainda que técnicas recentes de reabilitação de problemas neurológicos começam a incluir exercícios aeróbios e circuit training no tratamento dessas pessoas. A prática de atividades físicas tem ocasionado alguns benefícios motores para indivíduos com DP, embora seja percebida um pouco mais devagar que em indivíduos saudáveis ${ }^{17}$.

Em um estudo com 17 pacientes portadores da DP, comparam os efeitos do treadmill com apoio progressivo do peso do corpo com o método convencional de tratamento de distúrbios da marcha através de facilitação neuromuscular proprioceptiva (FNP) e uma intervenção controle ${ }^{17}$. Neste estudo é comprovada uma melhora significativa na velocidade $\mathrm{e}$ comprimento do passo depois do treadmill, mostrando uma redução relevante na fase de duplo apoio da marcha e nenhuma mudança significativa depois do FNP tradicional ou no grupo controle. Demonstrando com isso que esta atividade física pode ser um fator importante para evolução da marcha.

Em um trabalho com 15 pessoas com Doença de Parkinson, foram averiguados os efeitos de um treinamento com sobrecarga no equilíbrio e na resistência muscular, encontram efeitos positivos, destacando aumento da latência antes da queda em $15 \%$, redução em $20 \%$ a incidência de quedas, efeitos esses que permaneceram inalterados por ao menos 4 semanas depois do término do treinamento ${ }^{18}$. O treinamento ainda melhorou a capacidade de manter o equilíbrio durante condições de instabilidade. A resistência muscular dos ísquios tibiais, quadríceps e gastrocnêmico aumentou, sendo este último o que mais apresentaram melhoras, esses músculos se mantiveram mais vigorosos que no início do treinamento, mesmo que os participantes não tendo se mantido em treinamento ${ }^{18}$. Os autores ainda destacam que esses ganhos são importantes para conservar a capacidade funcional e prevenir lesões por quedas.

Um estudo onde se submete pacientes de Parkinson a caminhadas diárias nas montanhas, registrou-se melhoras gerais nestes pacientes, portanto a integração de pacientes em programas de exercício de baixa a moderada intensidade é uma intervenção efetiva para reduzir o declínio das capacidades funcionais ${ }^{13}$. Este autor acredita que a recuperação da performance física e as capacidades orgânicas fortalecidas aumentam a autoconfiança e auto-suficiência do paciente ajudando na reintegração dele à sociedade.

Em um estudo com indivíduos portadores da DP classificados como moderadamente ativos e sedentários, foi observado que os sedentários apresentavam níveis de $\mathrm{VO} 2$ máximo menores que indivíduos saudáveis da mesma faixa etária, enquanto que os moderadamente ativos alcançaram níveis de VO2 acima dos valores esperados como normal para pessoas com DP, mesmo assim continuavam apresentando a típica marcha do Parkinson (comprimento de passo reduzido e cadência elevada $)^{19}$. Estes resultados, segundo os autores sugerem que aqueles indivíduos com leve a moderada DP se desempenhar exercícios aeróbicos regularmente mantém normal sua capacidade cardiovascular apesar do déficit neurológico, o que se torna importante visto que o principal para as pessoas com DP é a manutenção da qualidade de vida (QV).

Outro ganho referido por pacientes com Parkinson, participantes de um programa de EF regular foi uma melhora significativa na memória recente, diminuição de náuseas e menor incontinência urinária e retenção de líquidos ${ }^{20}$.

Exercícios modificam o funcionamento do cérebro, mas os mecanismos de como isso acontece ainda são desconhecidos, estudos sugerem que isso ocorre porque o EF intenso disponibiliza cerca de 7 a 18\% a mais que o grupo controle (sem exercício), íons cálcio $\left(\mathrm{Ca}^{2+}\right)$ para o cérebro, essa oferta de $\mathrm{Ca}^{2+}$ estimula a síntese de dopamina ${ }^{20}$. O nível de dopamina no neostriatum e núcleo caudado, em ratos submetidos a $\mathrm{EF}$ intenso por 120 minutos, estiveram respectivamente $31 \%$ e $28 \%$ mais elevado que no grupo controle. A perda dopaminérgica no striatum é um dos maiores sintomas da DP e o tratamento mais freqüente é a reposição através do L-DOPA, o percussor imediato da dopamina. Assim sendo, alguns sintomas da DP podem ser retificados pelo $\mathrm{EF}^{20}$. 
Foram encontrados mais efeitos benéficos na combinação de EF e o programa de medicamentos do que com a terapia medicamentosa unicamente. $\mathrm{E}$ também aponta a AF como uma tendência de adaptação para tratamentos convencionais ${ }^{13}$.

Observaram que o EF intenso provoca um pequeno, no entanto significativo aumento do GDNF no striatum, hormônio esse que tem sido especulado como "fator de sobrevivência", ou seja, tem papel protetor contra substâncias danosas aos neurônios dopaminérgicos ${ }^{14}$. Na presença dele os neurônios dopaminérgicos aumentam de tamanho e apresentam processos mais longos formando uma rede mais densa de dendritos e axônios que células não expostas ao fator.

O treinamento e a reabilitação com o uso de sobrecarga podem estimular uma plasticidade no cérebro e na medula espinhal e até mesmo a neurogêneses, melhorando os resultados do comportamento, assim como, o treinamento sensório motor intenso parece funcionar de maneira "neuroprotetora", durante o processo de degeneração lenta dos neurônios da $\mathrm{SNc}^{18}$.

As evidências sugerem que reparos a danos no cérebro podem ser promovidos através de uma variedade de experiências incluindo a atividade motora $^{14}$.

Pessoas com DP deveriam ser encorajadas a manterem-se ativas, principalmente para o desempenho de atividades aeróbicas buscando assegurar a capacidade de realizar algumas tarefas ${ }^{19}$. Estes mesmos autores observam que os indivíduos com DP decrescem seu nível de AF significativamente mais rápido do que pessoas não doentes da mesma idade, provavelmente em virtude dos problemas motores como a acinesia e as dificuldades na marcha e outros, no entanto os que se mantém fisicamente ativos permanecem por mais tempo independentes.

De maneira geral pode-se dizer que o EF não cura o paciente com Parkinson, mas evita o agravamento de uma série de sintomas que dificultam a sua vida, como: diminuição do torque articular, através da redução do tônus e da resistência muscular; redução da incapacidade de realizar algumas tarefas devido a rigidez muscular e a acinesia podendo melhorar a coordenação motora afetada pelo tremor parkinsoniano; recuperação muscular; amenizar disfunções na marcha e no equilíbrio; aliviar os efeitos da bradicinesia; manter a independência funcional do indivíduo e reintegrá-lo a sociedade.
Devido a escassez de estudos enfocando os efeitos dos EF na DP há a necessidade de realização de outros estudos enfocando os efeitos dos diferentes tipos de exercícios físicos (aeróbicos e anaeróbicos), na busca da ampliação dos conhecimentos a respeito de medidas terapêuticas opcionais aos indivíduos que desejam amenizar os efeitos/sintomas da DP.

\section{CONCLUSÕES}

Parece haver uma tendência em acreditar que o exercício físico regular, principalmente o aeróbico, é benéfico para pacientes com DP, pois reduz sintomas como a hipocinesia, bradicinesia, distúrbios da marcha, degeneração neuronal, sendo então reconhecido como um meio auxiliar às terapias tradicionais (medicamentosa).

\section{REFERÊNCIAS BIBLIOGRÁFICAS}

1. Dmochowski R. Female Voiding Dysfunction and Movement Disorders. Inter UrogynecolJ 1999; 10: 144-151.

2. Rossi B, Siciliano G, Carboncini C, Manca ML, Massetani R, Viacava $\mathrm{E}$, et al. Muscle modifications in Parkinson's disease: myoelectric manifestations. Electroencephalogrclin Neurophysiol 1996; 101: 211-218.

3. Juh R, Kim J, Moon D, Choe B, Suh T. Different metabolic patterns analysis of Parkinsonism on the 18 F-FDG PET. Eur J Radiol 2004; 51: 223-233.

4. Morris ME, Iansek R. Characteristics of motor disturbance in Parkinson's disease and strategies for movement rehabilitation. Hum Mov Sci 1996; 15: 649-669.

5. Yarrow K, Brown P, Gresty MA, Bronstein AM. Force platform recordings in the diagnosis of primary orthostatic Tremor. Gait Post 2001; 13: $27-34$

6. Larumbe RI, Ferrer Valls JV, Viñes JJR, Guerrero D, Fraile P. Estudio casos-control de marcadores de estrés oxidativo y metabolismo del hierro plasmático en la enfermedad de parkinson. Rev Esp Salud Púb 2001; 75: 43-54.

7. Dickson JM, Grünewald RA. Somatic symptom progression in idiopathic Parkinson's disease. Parkinsonism and Related Disorders 2004.

8. Lukhanina EP, Kapoustina MT, Karaban IN. A quantitative surface electromyogram analysis for diagnosis and therapy control in Parkinson's disease. Park Rel Disord 2000; 6: 77-86.

9. Lou J, Benice T, Kearns G, Sexton G, Nutt J. Levodopa normalizes exercise related cortico-motoneuron excitability abnormalities in Parkinson's disease. Clin Neurophysiol 2003; 114: 930-937.

10. Nuyens G, De Weerdt W, Dom R, Nieuwboer A, Spaepen A. Torque variations during repeated passive isokinetic movements of the knee in subjects with Parkinson's disease and healthy control subjects. Park Rel Disord 2000; 6: 87-93.

11. Smiley-Oyen AL, Cheng HK, Latt LD, Redfern MS. Adaptation of vibration-induced postural sway in individuals with Parkinson's disease. Gait Post 2002; 16: 188-197.

12. Müller V, Lutzenberger W, Pulvermüller F, Mohr B, Birbaumer N. Investigation of brain dynamics in Parkinson's disease by methods derived from nonlinear dynamics. Exp Brain Res 2001; 137:103-110.

13. LökkJ. The effects of mountain exercise in Parkinsonian persons - a preliminary study. ArchGerontolGeriatr 2000; 31: 19-25.

14. Smith AD, Zigmond MJ. Can the brain be protected through exercise? Lessons from an animal model of parkinsonism. ExpNeurol 2003; 184: 31-39.

15. Miyai I, Fujimoto Y, Ueda Y, Yamamoto H, Nozaki S, Saito T, et al. Treadmill Training With Body Weight Support: Its Effect on Parkinson's Disease. Arch Phys Med Rehabil 2000; 81:849-852. 
16. Miyai I, Fujimoto Y, Ueda Y, Yamamoto H, Nozaki S, Saito T, et al. Long-Term Effect of Body Weight-Supported Treadmill Training in Parkinson's Disease: A Randomized Controlled Trial. Arch Phys Med Rehabil 2002; 83:1370-1373.

17. Pohl M, Rockstroh G, Rückriem S, Mrass G, Mehrholz J. Immediate Effects of Speed-Dependent Treadmill Training on Gait Parameters in Early Parkinson's Disease. Arch Phys Med Rehabil 2003;84(12):1760-1766.
18. Hirsch MA, Toole T, Maitland GG, Rider RA. The Effects of Balance Training and High-Intensity Resistance Training on Persons With Idiopathic Parkinson's Disease. Arch Phys Med Rehabil 2003; 84: 1109-1117. 19. Canning GG, Alison JA, Allen NE, Groeller H. Parkinson's Disease: An Investigation of Exercise Capacity, Respiratory Function, and Gait. Arch Phys Med Rehabil 1997; 78: 199-207.

20. Sutoo C, Akiyama K. Regulation of brain function by exercise. Neurobiol Dis 2003; 13: 1-14. 